\title{
Proposal of a Method to Detect Position of Moving Object from a Camera Using LEDs and Markers
}

\author{
Takato Komori, Shiyuan Yang* \\ Department of Electrical and Electronic Engineering, Kyushu Institute of Technology, Kitakyushu, Japan \\ *Corresponding Author: yang@elcs.kyutech.ac.jp
}

\begin{abstract}
The authors proposed a new indoor position detection method using a surveillance camera and LED markers for the increasing demand of robots. Robots such as automatic guided vehicles can be expected to be used not only in factories but also in a wide range of fields, so it is necessary to provide position detection system with easy introduction and high accuracy. The proposed method uses a general surveillance camera to capture a moving target with an LED marker. By using the information from camera image and the angles and height of the camera, a high accuracy position detection can be achieved when the height of the target LED maker can be obtained.
\end{abstract}

Keywords: Position detection, indoor, moving object, LED marker, robot, surveillance camera.

\section{Introduction}

In recent years, automatic guided vehicles such as robots have been widely used for transporting raw materials, parts, finished products, etc. at manufacturing sites such as automobile manufacturing factories. It is also widely introduced in non-manufacturing fields such as distribution centers that store and ship products. It is expected that the robot market will continue to expand in the future.

An accurate self-position estimation method is required when using an automated guided vehicle especially for indoor. Simultaneous Localization and Mapping (SLAM) technology is widely used by combining with various sensors such as Lidar, camera, acceleration sensor, wheel odometry, etc.

Lidar irradiates a laser beam, measures the time it takes for it to hit an object and bounces off, so that measures the distance and direction to the object. As a feature, the luminous flux density is higher than that of radio waves, and the position and shape can be detected with high accuracy. However, there is also the problem that it is difficult to detect objects that have low reflectance and it is expensive than other sensors.

Stereo camera method of estimating the distance information of an object contained in images taken by multiple cameras from the characteristics and positional relationship of the cameras. It is possible to detect objects at any distance, and it can also be used for objects with low reflectance. However, at least two cameras are required, and there are problems such as low spatial resolution and difficulty in detecting smooth and curved objects.

We propose a method only use one camera and an LED marker which is easy constructed and cheaper in the next chapter.

\section{Construction of Proposed Position Detection Method}

In this section, we show the construction for indoor position detection using a camera and an LED marker which is illustrated in Fig.1. Supposing the floor is a horizontal plane and installing the camera parallel to the horizontal plane. The LED marker blinks at a constant low frequency which can be detected by the camera.

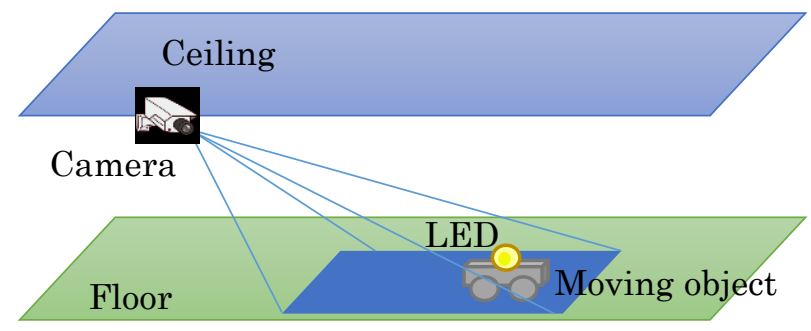

Fig. 1 Illustrate of one camera and LED marker position detection system 


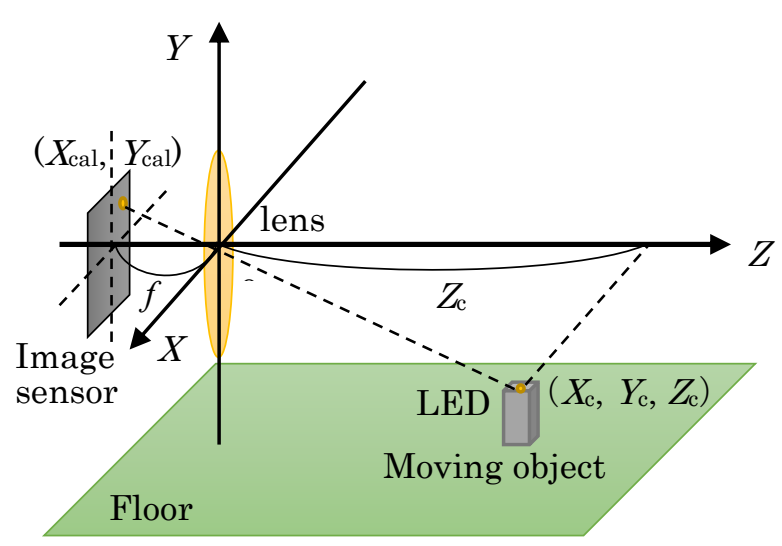

Fig. 2 Coordinates of one camera and LED marker position detection system

For convenience purposes, we set the coordinates of the measuring system as shown in Fig. 2. The center of the coordinates is set at the center of camera lens, so that the coordinates of the LED marker at horizontal plane are same to the corresponding $\mathrm{X}$ and $\mathrm{Z}$. Then we have

$$
\begin{gathered}
\frac{X_{c a l}}{f}=-\frac{X_{c}}{Z_{c}} \\
\frac{Y_{c a l}}{f}=-\frac{Y_{c}}{Z_{c}}
\end{gathered}
$$

where $X_{\mathrm{c}}, Y_{\mathrm{c}}, Z_{\mathrm{c}}$ are the coordinates of the LED marker, and $X_{\text {cal }}, Y_{\text {cal }}$ are the coordinates of the LED marker focused on the camera image sensor plane, respectively.

Let the heights of camera and LED marker be $H$ and $h$, respectively, which can be measured previously, we can define $H-h>0$ because a general surveillance camera is set at high position. Then we have $Y_{\mathrm{c}}=h-H$. Using this condition and combining the above Eqs. (1) and (2), we have the coordinates of the LED marker, $X_{\mathrm{c}}, Z_{\mathrm{c}}$ as follows:

$$
\begin{aligned}
& X_{c}=-\frac{(H-h) X_{c a l}}{Y_{c a l}} \\
& Z_{c}=\frac{(H-h) f}{Y_{c a l}}
\end{aligned}
$$

The coordinates of the LED marker focused on the camera image sensor plane $X_{\text {cal }}, Y_{\text {cal }}$ can be calculated from the pixel number $(m, n)$ which are the same directions to the $X$ and $Y$ axes in Fig. 2. That is

$$
X_{\text {cal }}=m \frac{X_{\text {camera }}}{M}-\frac{X_{\text {camera }}}{2}
$$

$$
Y_{\text {cal }}=n \frac{Y_{\text {camera }}}{N}-\frac{Y_{\text {camera }}}{2}
$$

where $M, N$ are the total number of camera image sensor and $X_{\text {camera, }} Y_{\text {camera }}$ are the sizes of camera image sensor in the horizontal vertical directions. Therefore Eqs. (3) and (4) become

$$
\begin{aligned}
X_{c} & =-\frac{(H-h)}{Y_{\text {cal }}}\left(m \frac{X_{\text {camera }}}{M}-\frac{X_{\text {camera }}}{2}\right) \\
Z_{c} & =\frac{(H-h) f}{n \frac{Y_{\text {camera }}}{N}-\frac{Y_{\text {camera }}}{2}}
\end{aligned}
$$

Equations (7) and (8) are the coordinates of the LED marker on the floor as shown in Fig. 2.

Next we show the process of coordinate measurement of LED marker.

Step 1:

Input the image from the camera to a computer and binarize the image using a general binarizing algorithm to extract the area of LED maker.

Step 2:

Calculate the center of LED marker with Morphological processing to get the center pixel number $(m, n)$.

Step 3:

Use Eqs. (7) and (8) to obtain the position of LED marker.

Step 4:

Get the next image from camera and repeat these steps to detect the position of LED marker with real time.

Figure 3 shows the flowchart of LED marker position detection. By repeating the detection of LED marker position, the position of a moving object can be measured at real time.

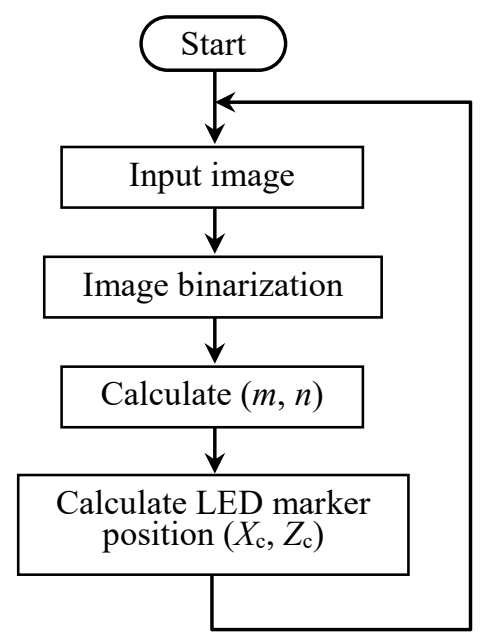

Fig. 3 Flowchart of LED marker position detection 


\section{Experiments and Discussions}

We show some experimental results using a simple construction with a surveillance camera (Panasonic network camera BB-SW174WA) and an LED light as the maker. The camera sensor sizes are $3.52 \mathrm{~mm}$ in horizontal direction and $2.64 \mathrm{~mm}$ in vertical direction. The image pixel numbers used in experiments are 640 pixels in Horizontal direction) and 480 pixels in Vertical direction. The camera focus distance is $1.95 \mathrm{~mm}$. The center height of camera sensor is set at $850 \mathrm{~mm}$ and center height of LED light is set at $200 \mathrm{~mm}$. Experiments were performed with same intervals of $50 \mathrm{~mm}$ in $Z$-axis direction and $X$-axis direction that the LED light was moved in the areas from $1500 \mathrm{~mm}$ to $2000 \mathrm{~mm}$ in $Z$-axis direction and from $-1000 \mathrm{~mm}$ to $1000 \mathrm{~mm}$ in $X$-axis direction. The vertical distance between the camera and the LED light was set as $700 \mathrm{~mm}$.

Figure 5 shows the experimental results of LED light maker at different coordinates on the vertical plane. The measured $X$-axis direction coordinate values are mostly near to the true value for various distance $Z$. The maximum of errors in $X$-axis direction was $50 \mathrm{~mm}$.

Figure 6 shows the measured $Z$-axis direction coordinates when the $X$-axis direction coordinate is changed with different $Z$ value. The measured $Z$-axis direction coordinate values are mostly near to the true value for various distance $X$. The maximum of errors in $Z$-axis direction was $30 \mathrm{~mm}$.

From the experimental results performed with poor set up as shown in Fig. 4, we obtained position detection with errors less than $50 \mathrm{~mm}$. This is enough for general usage such as indoor robot guiding and other moving object position detection. Moreover, it would be possible to reduce the errors by using high resolution camera. It is also possible to achieve high accuracy of measurement by using collimation to improve the measurement setup.

\section{Conclusions}

We proposed a new position detection method for the coordinate measurement of indoor moving object. This new method has the merit that a general surveillance camera can be utilized and a cheap LED marker can be used for easy image processing. It has an enough accuracy for indoor moving object detection purpose. In addition, this system can be combined to other purpose applications with position information supplying. In this study, we only considered the case that the camera is paralleled to the horizontal floor plane.
We will show analysis and experiments for other cases such as using the pan and tilt angles of camera in the near future.

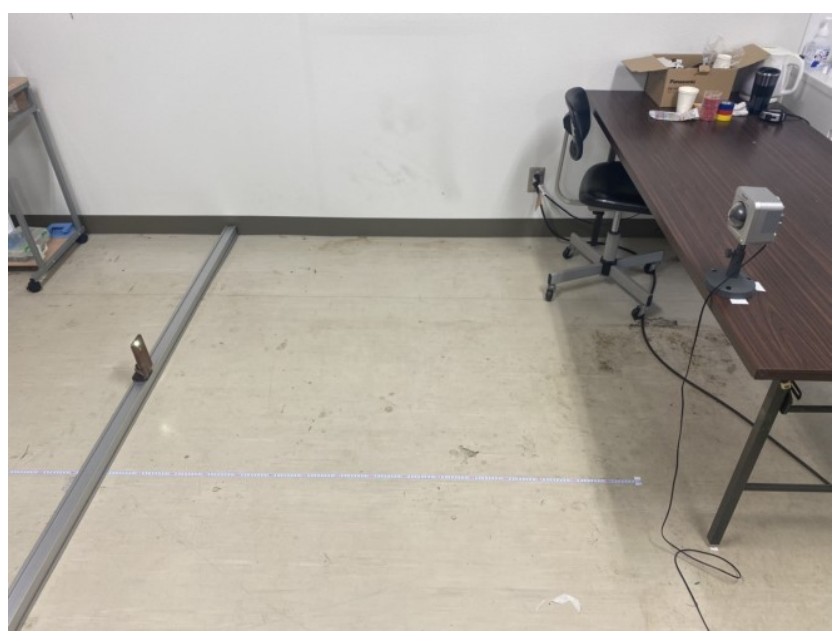

Fig. 4 Image of experimental construction

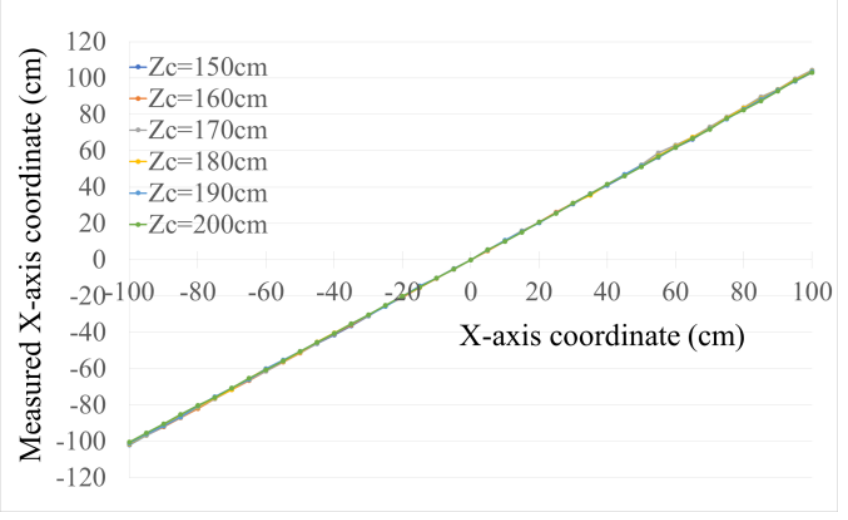

Fig. 5 Measurement results on $X$-axis with different $Z$ value

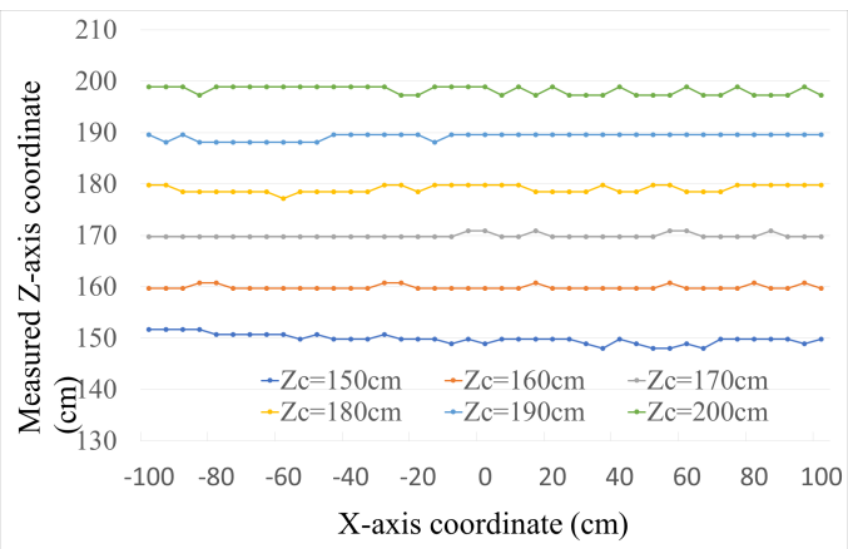

Fig. 6 Measurement results on $Z$-axis when the $X$-axis coordinate is changed with different $Z$ value 


\section{References}

(1) Hakan Koyuncu, Shuang Hua Yang : "A Survey of Indoor Positioning and Object Locating Systems", IJCSNS International Journal of Computer Science and Network Security, Vol. 10 No. 5, pp. 121-128, 2010

(2) Hisato Kawaji, Koki Hatada, Toshihiko Yamasaki, Kiyoharu Aizawa : "Image-based Indoor Positioning System: Fast Image Matching using Omnidirectional Panoramic Images", Proceedings of the 1st ACM international workshop on Multimodal pervasive video analysis, pp. 1-4, 2010

(3) Jayakanth Kunhoth, AbdelGhani Karkar, Somaya Al-Maadeed and Abdulla Al-Ali : "Indoor positioning and wayfinding systems: a survey", Human-Centric Computing and Information Sciences, pp. 1-41, 2020

(4) P. Bahl, and V. N. Padmanabhan, "RADAR: an inbuilding RF-based user location and tracking system", in Proceedings of IEEE INFOCOM, Vol. 2, pp. 775-784, 2000 